\title{
ARCHIMÈDE N'3
}

\section{DOSSIER THÉMATIQUE : DES FOSSÉS ET DES REMPARTS. ENCEINTES ET SITES FORTIFIÉS DU RHIN SUPÉRIEUR ENTRE PROTOHISTOIRE ET MOYEN ÂGE}

\section{Olivier BUCHSENSCHUTZ}

Avant-propos. Des enceintes en terre anhistoriques à Google Earth

8 Lizzie SCHOLTUS

Histoire de la recherche dans le bassin de Saint-Dié-des-Vosges

20 Maxime WALTER

Les sites de hauteur du massif vosgien. Actualisation des données et modalités d'implantation

37 Jean-Jacques SCHWIEN

Chateaux et enceintes des Vosges du Nord. Topographie et longue durée

49 Anne-Marie ADAM

La palissade dans tous ses états : I'enclos du Britzgyberg (Illfurth, Haut-Rhin) et autres aménagements palissadés dans les habitats du premier âge du Fer

60 Clément FÉLIU

L'enceinte inférieure du Frankenbourg (67) et les remparts à poteaux frontaux de la fin de l'âge du Fer dans l'espace du Rhin supérieur. Pour une révision de la typologie des Pfostenschlitzmauern

74 Jacky KOCH et Thomas FISCHBACH

Enceintes de hauteur en pierres et formes « primitives » de châteaux ? L'exemple du Bernstein

87 Adrien VUILLEMIN

Les enceintes urbaines en moyenne Alsace (1200-1850)

102 Jean-François PININGRE

Les enceintes de l'âge du Bronze et du premier âge du Fer en Franche-Comté. Un bilan des recherches

124 Clément FÉLIU et Jean-Jacques SCHWIEN

Conclusion. Nouvelles perspectives sur les enceintes du Rhin supérieur

\section{ACTUALITÉ DE LA RECHERCHE : ARCHÉOLOGIE DES RÉSEAUX}

\section{Claire CAMBERLEIN}

Les réseaux en archéologie : approche historiographique et interdisciplinaire

135 Thomas HUTIN

Lieux d'échanges et espaces publics en Gaule à La Tène finale

150 Streeve GENTNER

Économie du fer et voies de communication, de l'abattage du minerai à la distribution

du métal : I'exemple du nord de la Forêt-Noire au Ve siècle av. J.-C.

169 Loup BERNARD et Rémy WASSONG

Du Danemark au Fossé rhénan. Un siècle d'analyse des voies de communications protohistoriques : évolution des méthodes et mise en commun des données

184 Steeve GENTNER et Rémy WASSONG

Conclusion. L'archéologie des réseaux : une thématique aux multiples facettes

\section{VARIA}

187 Fábio VERGARA CERQUEIRA

To march in phalanx, to jump with weights, to tread the grapes, to knead the bread. What is the aulos for?

206 Hermann AMON

Les supra-commandements comme solution à la crise militaire du III siècle de l'Empire romain sous Philippe l'Arabe et Gallien

218 Martina BONO

Il processo di Cremuzio Cordo in Dio LVII, 24, 2-4

\section{LA CHRONIQUE D'ARCHIMÈDE}

228 Frédéric COLIN (éd.)

La Chronique d'Archimède. Bilan des activités scientifiques 2015-2016 de I'unité mixte de recherche 7044 


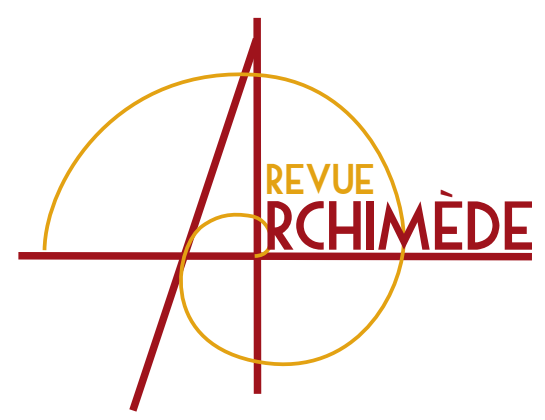

\title{
CONCLUSION. NOUVELLES PERSPECTIVES SUR LES ENCEINTES DU RHIN SUPÉRIEUR
}

\author{
Jean-Jacques SCHWIEN \\ Maître de conférences en archéologie médiévale \\ Université de Strasbourg \\ UMR 7044 Archimède \\ schwien@unistra.fr
}

Clément FÉLIU

Responsable d'opération Inrap Grand-Est sud UMR 7044 Archimède

clement.feliu@inrap.fr
L'ensemble des contributions réunies ici reflète bien le travail d'équipe engagé depuis 2013. L'équipe d'Archimède qui mène cette réflexion collective sur les enceintes regroupe une vingtaine de personnes, chercheurs de I'UMR, chercheurs associés et doctorants, issus de diverses institutions : Université de Strasbourg, Service régional de l'Archéologie, Pôle archéologique interdépartemental rhénan, Institut national de recherches archéologiques préventives. Son projet s'organise autour de plusieurs axes complémentaires d'étude du paysage fortifié, sous tous ses aspects, entre Vosges et Forêt-Noire, depuis le coude du Rhin et les premiers contreforts du Jura jusqu'à la région de Lauterbourg et Karlsruhe, tout en jetant un regard sur les régions voisines, où les recherches sont plus avancées, comme la Franche-Comté, par exemple (Jean-François Piningre).
Son champ chronologique s'étire de la Protohistoire au Moyen Âge, la prise en compte d'une résolution chronologique longue offrant le moyen d'étudier les évolutions des fortifications à différentes périodes qui ne présentent à première vue que peu de points communs, mais qui révèlent, à l'analyse, des dynamiques semblables : on aura noté, toutefois, que l'essentiel des travaux en cours privilégie de fait la Protohistoire et le Moyen Âge. Quant à la nature des sites, après mûre réflexion, le choix a finalement porté sur les enceintes fortifiées collectives, y compris des villes de la fin du Moyen Âge, intégrant les fortifications non datées (afin de pouvoir éventuellement les attribuer à l'une ou l'autre période) mais excluant les fermes et enclos fossoyés protohistoriques ou encore les forteresses adaptées aux armes à feu d'époque moderne. 
Les moyens mis en œuvre relèvent à la fois des ressources classiques de la carte archéologique (recherches documentaires, analyse de cartes) et des travaux de terrain. Les premières n'apparaissent ici que de façon indirecte, l'essentiel appartenant à un acquis plus ou moins ancien et déjà publié. On notera toutefois la reprise de l'ensemble des données disponibles sur la fortification de La Bure dans le bassin de Saint-Dié (Lizzie Scholtus) qui offre les bases solides d'un nouveau programme de terrain, en cours de développement. En filigrane, il faut souligner l'apport des outils numériques, facilitant l'accès à des ressources longtemps éparses (on pense aux cartes anciennes) mais aussi et surtout des images Lidar : la couverture systématique du département du Haut-Rhin, accessible librement sur le site web de la collectivité fournit ainsi un catalogue de sites potentiels sous couvert forestier (et généralement de hauteur) sans commune mesure avec ce que les travaux anciens ont pu livrer [1].

Mais c'est avant tout par les travaux de terrain que le dossier est en cours de renouvellement. Des prospections systématiques sur les enceintes de hauteur rurales non datées ont été engagées dans le cadre de deux masters (un par département), dont le premier, déjà soutenu est évoqué ici (Maxime Walter) : ces prospections permettent à la fois de revoir des analyses anciennes à l'aide des données des recherches récentes, mais aussi d'éliminer des sites aux indices d'occupation mal cernés. Entamées dans un autre contexte que notre projet mais dûment intégrées depuis, deux thèses portant sur l'Alsace centrale livrent un catalogue de deux types d'enceintes médiévales désormais bien datées par le truchement des données de prospection, des relevés, des documents d'archives, des analyses diverses : les proto-châteaux (Jacky Koch) et les villes petites à moyennes (Adrien Vuillemin). Enfin, I'organisation de fouilles, soit pour des enceintes déjà caractérisées au travers de sondages (Britzgyberg, A.-M. Adam) ou découvertes au cours de prospections (Frankenbourg, Cl. Féliu), venant en complément d'opérations déjà publiées (Heidenstadt, Fossé des Pandours, Koestlach, Hohlandsbourg), apporte évidemment des données incomparables sur les modalités constructives et la chronologie des sites protohistoriques.

Il est trop tôt évidemment pour dresser un bilan complet de l'ensemble des travaux engagés, sur la nature des sites, leur répartition spatiale, les choix topographiques ou architecturaux, les grandes scansions chronologiques; toutefois, certaines pistes se profilent déjà. En termes d'espace, il apparaît ainsi que des zones de fortes densités, autour de Saverne, dans les Vosges du Nord ou sur le piémont d'Alsace centrale, côtoient des secteurs nettement plus vides, dans la région de Bouxwiller ou les vallées de Thann ou Masevaux (Maxime Walter). Cette sectorisation générale semble perdurer sur la large échelle chronologique envisagée de la Protohistoire au Moyen Âge, bien que les lieux soient assez rarement réoccupés d'une période à l'autre, à l'exception notable de la Hohlandsbourg ou du Frankenbourg, par exemple. Cette géographie persistante amène à se poser la question du choix des lieux d'implantation sur la très longue durée, indépendamment des organisations socio-politiques (Jean-Jacques Schwien) : déterminisme géographique, importance des communications, accès aux ressources... Il manque ici des données plus fermes sur la nature, le nombre et l'organisation territoriale des enceintes de I'Antiquité tardive pour mieux évaluer les ruptures et continuités entre Préhistoire et Moyen Âge.

À ces premiers résultats sur les modalités d'implantation des sites viennent s'ajouter de nouveaux développements sur les techniques architecturales mises en œuvre dans la construction des systèmes fortifiés et sur leur rôle dans les sociétés anciennes. Le renouvellement des données disponibles sur les fortifications protohistoriques, au Britzgyberg (Anne-Marie Adam) ou au Frankenbourg (Clément Féliu), permet de questionner les typologies établies de longue date pour les remparts de l'âge du Fer. Il offre aussi l'opportunité de proposer de nouvelles interprétations sur la fonction de ces remparts, dont le caractère défensif ne peut plus être le seul mis en avant. Les résultats de la fouille de l'enceinte en pierres sèches du Bernstein invitent pour leur part à la prudence quant à I'analyse des matériaux et techniques architecturales comme critère chronologique, puisqu'ici, la mise en œuvre la plus fruste n'appartient pas forcément à la phase la plus ancienne, mais au contraire à l'une des plus récentes (Thomas Fischbach).

En termes de chronologie, il se confirme maintenant que notre région, comme d'autres, est marquée par plusieurs vagues successives d'occupation des enceintes de hauteur : à une première phase de fortification à la fin du premier âge du Fer et au début du second succède, sans continuité directe, un deuxième temps fort à la fin de La Tène, avec l'aménagement de quelques sites de très grandes dimensions à côté d'enceintes plus petites [2], puis un troisième au Bas-Empire et au cours du premier Moyen Âge, qui voient le réaménagement d'enceintes « collectives » [3]. De ce point de vue, la datation (de la construction ou du réaménagement ?) de l'enceinte du

[1] Cette remarque anticipe la présentation des résultats réunis dans le master en cours depuis 2014 d'Emmanuelle Wittlin, avec une quarantaine de sites recensés.

[2] FICHTL 2003.

[3] Steuer \& Bierbrauer 2008 ; Bourgeois 2006, p. 113-142. 
mont Sainte-Odile vers 650 ap. J.-C. est évidemment emblématique [4], mais c'est l'analyse des enceintes « tardives », au moment du passage vers le château féodal qui est ici plus pertinente puisqu'elle pointe un phénomène qui ne concerne pas que des résidences princières ou de haute époque (Jacky Koch). On peut inscrire I'étude des villes, petites et moyennes, dans cette même veine générale : plutôt que de voir seulement une continuité entre enceintes et châteaux, il faut envisager la succession entre les enceintes « collectives » du premier Moyen Âge et les villes plus tardives (Adrien Vuillemin). Les secondes apparaissent en effet alors que les premières disparaissent et connaîtront une durée de vie réelle plus longue que le château [5]. Si des avancées peuvent être soulignées pour les périodes les plus récentes, d'autres phases chronologiques doivent encore faire l'objet de recherches qui permettront de qualifier de manière plus précise leurs rapports aux fortifications : pour l'âge du Bronze, par exemple, I'Alsace ne compte que des traces éparses de fréquentation sur les hauteurs, à l'exception notable du Hohlandsbourg et du Mont Sainte-Odile.
On peut également noter que le dossier des enceintes de I'Antiquité tardive n'a pas été repris depuis une maîtrise en 1991 [6], à laquelle il serait possible d'ajouter les résultats des recherches les plus récentes.

La prise en compte d'une résolution temporelle large, de la Protohistoire au Moyen Âge, offre de nouvelles clés de compréhension de ces objets singuliers que sont les enceintes. Elle permet en effet de les replacer dans leur contexte chronologique spécifique, puis de comparer les paysages fortifiés ainsi décrits pour chacune des périodes successives. Tout en prenant garde aux anachronismes, les analogies et différences de quelque nature que ce soit - topographiques, architecturales, morphologiques, chronologiques, etc. - peuvent être interrogées afin de rendre à ces sites la complexité sociale, politique ou économique qui était la leur.

[4] Steuer 2012, p. 27-69.

[5] Outre la thèse d'A. Vuillemin, encore inédite, on verra aussi avec profit HeNIGFELD \& MASQUILIER 2008.

[6] SCHWien 2015, p. 214-222; KUnHLE 1991.

Bourgeois, Luc, 2006, «Les résidences des élites et les fortifications du haut Moyen Âge en France et en Belgique dans leur cadre européen: aperçu historiographique (1995-2005) », Cahiers de Civilisation Médiévale 46, p. 113-142.

FichtL, Stephan, dir., 2003, « Les oppida du nord-est de la Gaule à La Tène finale », Archaeologia Mosellana 5, p. 7-238.

Henigfeld, Yves \& Masquilier, Amaury, dir., 2008, Archéologie des enceintes urbaines et leurs abords en Lorraine et en Alsace (XII ${ }^{e}-X V^{e}$ siècle), Dijon (Revue archéologique de l'Est, 26e supplément).

KunHLe, Gertrud, 1991, Les fortifications romaines de l'Antiquité Tardive des vallées du Rhin supérieur et du Haut-Rhin, mémoire de maîtrise, université de Strasbourg.

Steuer, Heiko, 2012, « Studien zum Odilienberg im Elsass », Zeitschrift für Archäologie des Mittelalters 40, p. 27-69.

Steuer, Heiko \& Bierbrauer, Volker, dir., 2008, Höhensiedlungen zwischen Antike und Mittelalter von den Ardennen bis zur Adria, Berlin.

Schwien, Jean-Jacques, 2015, « Les villes en Alsace entre I'an mil et les années 1200 », L'Alsace au cœur du Moyen Âge, Lyon, p. 214-222. 